\title{
Impact of the species compartment definition on quantitative modeling of microbial communities
}

\author{
Marko Budinich ${ }^{1}$, Jérémie Bourdon ${ }^{1} \&$ Damien Eveillard ${ }^{1}$ \\ ${ }^{1}$ LINA UMR 6241, Université de Nantes, EMN, CNRS, France
}

\begin{abstract}
R
ecent advances in genome-scale metabolic network reconstruction paved the way to the use of quantitative modelings such as FBA. However, despite the great interest of these techniques to tackle quantitative features, microbial community modeling remains unclear. Whereas studies represent a microbial community with several compartments for each microbial strains and their common pool, others advocate for the use of a single compartment that combines all reactions. Here we show that both modelings lead to different optimal quantitative solutions. This study illustrates this difference by the use of the flux module technique, that describes, in a compact way, the optimal solution space as computed by FBA-like techniques. For application, this paper computes the flux modules of a hot spring microbial community (represented by Synechococcus spp., Chloroflexus and Roseiflexus spp.) and a microbial methagenic system (Desulvovibrio vulgaris and Methanococcus maripaludis) sulfate reducing bacteria), while emphasizing the quantitative changes that occurs when one assumes either the consortium as a "single compartment" or a multiple compartment.
\end{abstract}

\section{Introduction}

Following the surge of high throughput experiments to investigate microbial ecosystems, network systems ecology techniques risen their interest by proposing a qualitative description of ecosystems. For instance, by focusing on "who is 
there and who is not" [13], these techniques emphasize networks of microbes that co-occurred. However, the functional understanding of these communities remains often challenging when techniques solely consider qualitative community description rather than introducing (partial) quantitative knowledge. Considering the metabolic network of a community is one way to overcome this weakness [21]. One assumes the microbial ecosystem behaviors driven by the metabolic reactions encoded by each microbial genomes. In other words, thanks to multiple metabolic enzymes, microbes interplay within the environment and promote a whole metabolic network responsible for quantitative behavior $[6,3]$. Once the metabolic network identified, constraint-based modelings are standardly used to reproduce quantitative properties of the whole metabolic network at a molecular resolution (see [11] or [18] for review)

However, whereas these modeling techniques such as FBA-like approaches became standard modeling routines for single cell systems, their applicative conditions remain to be deeper investigated when applied on microbial communities. In particular, microbial community metabolic network could be either reconstructed as a single integrated network by merging all reactions monitored by all bacterial strains (i.e. Single Cell Hypothesis - SCH), or considering natural boundaries between species by considering strain specific genes (i.e. Multiple Compartment Hypothesis - MCH) as already promoted in tissu specific networks $[2,10]$ or well-studied human gut microbial community [14]. Because both assumptions implies a distinct experimental efforts, this study proposes an analysis of $\mathrm{SCH}$ and $\mathrm{MCH}$ consequences on microbial community metabolic models. It is worth to notice herein that such an approach is a natural extension of a previous work of [9]. In 2010, Niels Klitgord and Daniel Segrè proposed a study of the impact of compartmentalization in metabolic flux models via the consideration of organelles within the metabolic network of yeast. However, at the time of the study, no genome-scale metabolic description of microbial community was available. We propose to overcome this weakness by taking benefits from recent biotechnological progresses. We complete the previous study by exploring herein the difference of both - $\mathrm{MCH}$ and $\mathrm{SCH}$ - hypotheses on two distinct genome-scale community metabolic networks. First, we analyze a microbial mat model that uses three microbial strains: Cyanobacteria, filamentous anoxygenic phototrophs and sulfate reducing bacterias [17], to analyze quantitative and qualitative differences in the predictions; secondly, we use Desulfovibrio vulgaris and Methanoccocus maripaludis microbial system, a classical example of syntrophic growth in anaerobic environment [16]. 


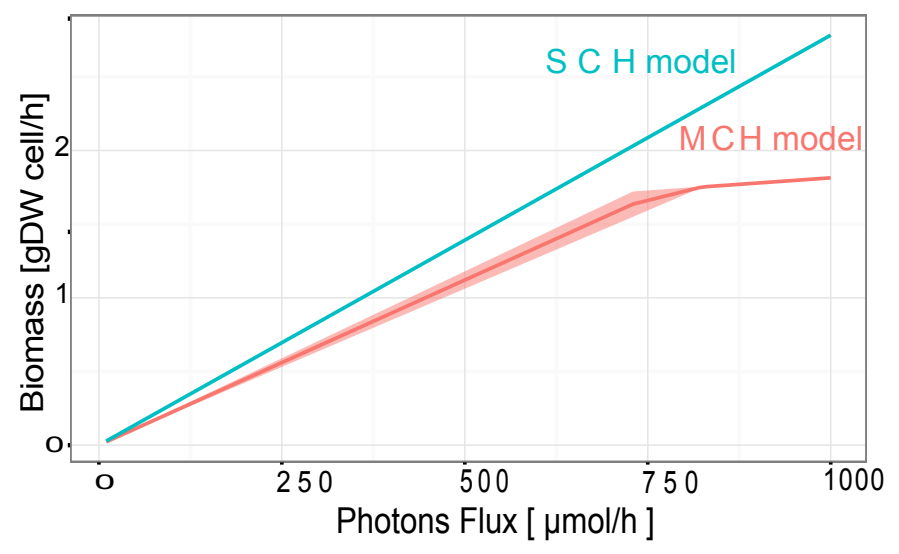

Figure 1: Quantitative simulations of Multiple Compartments Hypothesis (MCH) and Single Compartments Hypothesis (SCH) models of a hot spring microbial mat system. Flux variability simulation results show an increase of the whole community biomass under a range of light conditions. Simulations of $\mathrm{MCH}$ and $\mathrm{SCH}$ metabolic models are pictured in red and blue respectively.

\section{Results}

For the sake of application, one first considered the phototrophic microbial community system during day light composed Synechococcus spp., called SYN, filamentous anoxygenic phototrophs related to Chloroflexus and Roseiflexus spp., called FAP, and sulfate reducing bacteria (SRB) [17]. This community consumes $\mathrm{CO}_{2}$ and releases $\mathrm{O}_{2}$ by photosynthesis. As a byproduct of the rubisco activity, glycolate is produced by SYN, which will be later used as an organic substrate by FAP, along with acetate. Besides, SRB can consume organic compounds and reduce sulfate using $\mathrm{H}_{2}$. $\mathrm{MCH}$ community model describes a metabolic network for each strain as well as external metabolites such as $\mathrm{H}_{2}$, $\mathrm{O}_{2}, \mathrm{NH}_{3}$, glycogen and acetate (136 reactions) [17]. As a modeling contribution, a community biomass function was included to represent the ecosystem growth plus one extra reaction for preserving $\mathrm{O}_{2} / \mathrm{CO}_{2}$ ratio as used by rubisco. As reported in [17], the so-called "Pool model" represents the SCH community model (59 reactions: 48 core and 11 exchange reactions) - see Figure 2 and Supp for details.

Both MCH and SCH models reproduce previous results and are qualitatively consistant with available experiments [16, 22] (see Figure 1). Both models show similar range of biomass increase when light increase. Naively, these 
similar predictions may lead to over-interpret that both model are identical, which do not advocate for the use of $\mathrm{MCH}$ that is experimentally expensive. However, solely, these interpretations are not sufficient and might lead to misunderstandings. To overcome these shortcomings, one must indeed consider extensive simulations to investigate all solutions as provided by both SCH and $\mathrm{MCH}$ models, and not a unique optimal solution. Indeed, while constraintbased modelings describe sets of fluxes that go through all metabolic reactions at equilibrium, above simulations represent, among all fluxes, a unique flux combination that maximizes one given objective - herein the biomass production. More recent Flux Variability Analysis (FVA) improves this unique description by pinpointing all satisfying solutions over a wide range of environmental conditions. Promoting a systematic exploration of these solutions, Flux Module (FM) technique [12] analyzes how, among all solutions, some reactions are systematically correlated - emphasizing subnetworks that connect a subset of substrates and products [7]. These subnetworks or modules are unique and result from all potential quantitative solutions. Implicitly, different modules imply different quantitative predictions (mathematically called optimal solution space). From a biological viewpoint, its application on single cell organisms shows modules as a sensitive description of biological functions (see supplementary for E. coli modules).

$\mathrm{MCH}$ and $\mathrm{SCH}$ models produce distinct modules, which clearly emphasizes fundamental differences between $\mathrm{MCH}$ and $\mathrm{SCH}$ solutions. $\mathrm{SCH}$ shows only one module (purple in Figure 2), containing 31 reactions ( $52.2 \%$ of overall reactions): 20 reactions covered by $\mathrm{MCH}$ modules and 11 not previously highlighted. $7 \mathrm{MCH}$ module reactions do not belong to the SCH module. MCH SYN reactions are decoupled from other networks, confirming previous studies [17] that highlights SYN as a primary producer for all possible microbial interactions. Complementary, FAP and SRB are linked via acetate and $\mathrm{H}_{2}$ metabolisms. As additional differences, the first glycolysis phase (R1-R2) and pentose phosphate reactions (R5-R9) are connected in $\mathrm{SCH}$, which is not true when each organism is considered separately. SCH module is independent from uptake reactions; whereas MHC modules depict acetate processing of FAP and SRB linked to $\mathrm{O}_{2}$, $\mathrm{H}_{2}$ and $\mathrm{CO}_{2}$ exchanges.

For the sake of generalization, a similar modeling comparison was applied on a methanogenic microbial system composed of Desulfovibrio vulgaris and Methanococcus maripaludis. D. vulgaris uses lactate fermentation and sulfur reduction to gain energy, while producing gaseous hydrogen. M. maripaludis uses hydrogen to reduce $\mathrm{CO}_{2}$ into methane, which avoid the accumulation of $\mathrm{H}_{2}$ that might decrease the chemical energetic potential of $D$. vulgaris. The corresponding $\mathrm{MCH}$ model owns 243 reactions (respectively 145 and 97 reactions 
bioRxiv preprint doi: https://doi.org/10.1101/018010; this version posted April 15, 2015. The copyright holder for this preprint (which was not certified by peer review) is the author/funder, who has granted bioRxiv a license to display the preprint in perpetuity. It is made available under aCC-BY-NC-ND 4.0 International license.

MCH Model

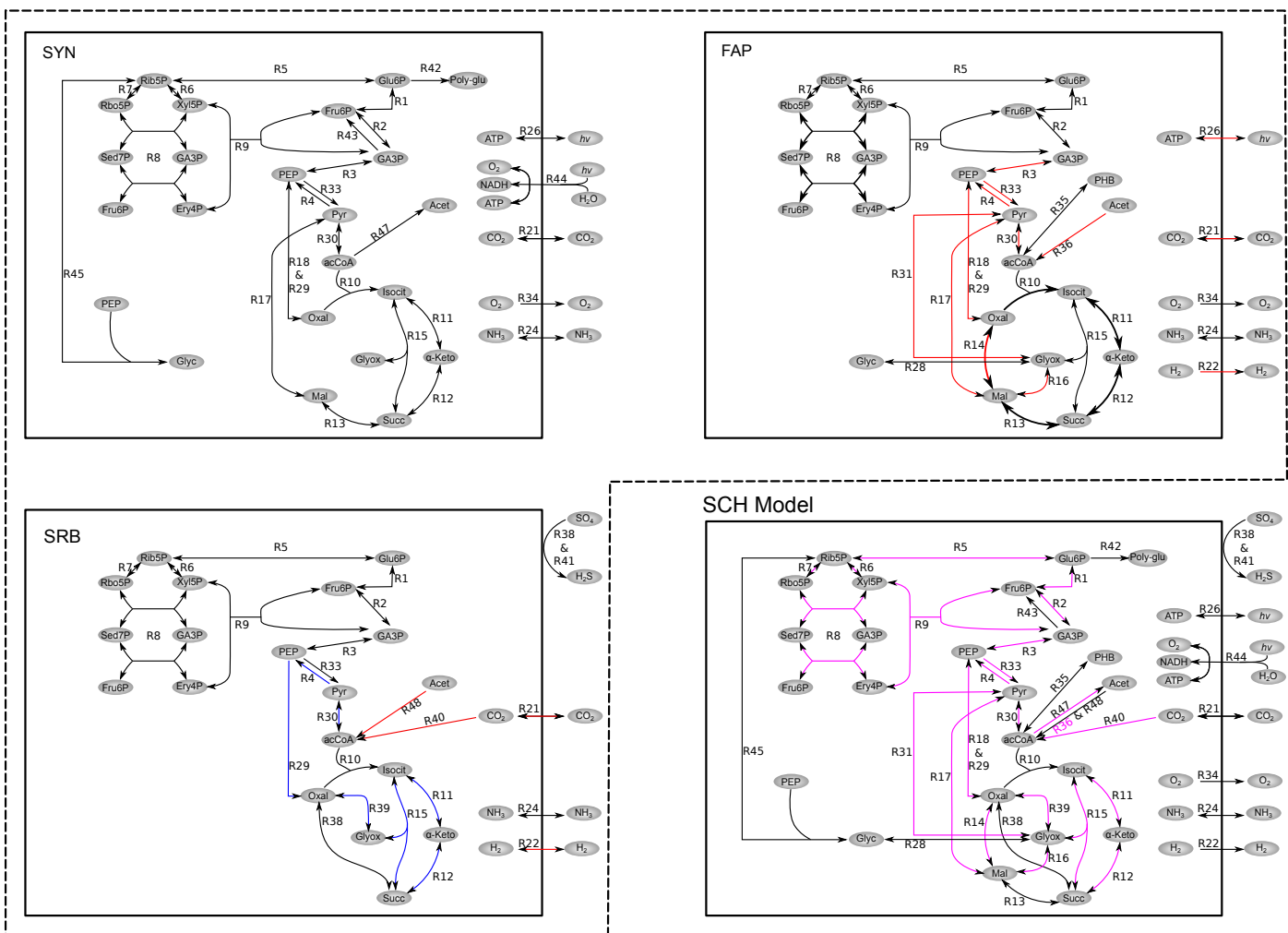

Figure 2: Description of metabolic networks related to the microbial mat community system and corresponding modules illustrations. SYN, FAP and SRB depict bacterial strains of the MCH metabolic model. SCH model represents the same metabolic system with no consideration of the compartments while conserving the naming convention of the MCH networks. For the sake of illustration exchange reactions between compartments are not shown. MCH model reveals 2 modules (26.5\% of the whole set of metabolic reactions). One module contains 28 reactions (red) that span through FAP and $S R B$, whereas another (blue) involves 8 reactions. Reactions of the $S C H$ module are depicted in purple. 
for D. vulgaris and M. maripaludis) and the $\mathrm{SCH}$ model is composed of 221 unique reactions, after deletion of redundant reactions. Again $\mathrm{MCH}$ and $\mathrm{SCH}$ modules are different (see Supplementary). Both models show a unique module: 124 reactions (48.6\% of $\mathrm{MCH}$ model) and 187 reactions ( $84.6 \%$ of SCH model). Reactions related to $\mathrm{H}_{2}$ and acetate transport are not related in $\mathrm{SCH}$ model, whereas they are in $\mathrm{MCH}$. Additionally, pentose phosphate cycle reactions of $D$. vulgaris and M. maripaludis are linked in $\mathrm{SCH}$ module but not in $\mathrm{MCH}$, which might lead to misunderstand bacterial interpretations.

\section{Discussions}

Despite similar quantitative simulations, this study shows significant differences between $\mathrm{SCH}$ and $\mathrm{MCH}$. However, this communication do not advocate for either of both modeling assumptions. Biologically, SCH models have been widely employed to study metabolite exchanges between species (e.g., cocultures [20][5] or species within a complex environment [8]), whereas MCH models have been used to describe microbial communities, where each member seeks to maximize their own biomass [19]. Both assumptions are equivalent when one is interested by predicting overall quantitative behaviors of a microbial community, which is mostly explained by similar exchange reactions between $\mathrm{SCH}$ and $\mathrm{MCH}$ models. A protocol driven by $\mathrm{SCH}$ might be mostly sufficient for overall predictions with non further functional investigations. Reversely, $\mathrm{MCH}$ driven protocols present a significant cost to decipher boundaries between species and origin of genes within a meta-genome [18], but appear as necessary to investigate fine quantitative interactions within the community.

From a methodological viewpoint, this study advocates for the use of Flux Modules to compare metabolic models. Modules represent an abstraction of all Flux Variability simulations for a given metabolic model. Indeed Flux Module technique is a natural way to resume the methodological work of [9] that proposes an extensive analysis of yeast metabolic flux estimation with and without compartmentalization. Since our study pinpoints similar conclusions to [9], both studies reinforces the need for further constraint-based modelings dedicated to multiple compartments simulations as motivated by [22, 23].

\section{Acknowledgments}

M.B. is supported by CNRS grant, M.B., J.B. and D.E. are supported by GRIOTE project. D.E. is supported by ANR grant SAMOSA. 


\section{References}

[1] Begon M, Townsend CR and Harper JL. (2006). Ecology: from individuals to ecosystems. Malden, MA: Blackwell Pub; 4, 2006.

[2] Bordbar A, Feist AM, Usaite-Black R, Woodcock J, Palsson BØ, Famili I. (2011). A multi-tissue type genome-scale metabolic network for analysis of whole-body systems physiology. BMC Syst Biol 5:180.

[3] Dittami SM, Eveillard D, Tonon T. (2014). A metabolic approach to study algal-bacterial interactions in changing environments. Mol Ecol 23:1656-1660.

[4] Follows MJ, Dutkiewicz S. (2011). Modeling diverse communities of marine microbes. Ann Rev Mar Sci 3:427-451.

[5] Hanly TJ, Henson MA. (2010). Dynamic flux balance modeling of microbial co-cultures for efficient batch fermentation of glucose and xylose mixtures. Biotechnology and Bioengineering 108:376385 .

[6] Karsenti E, Acinas SG, Bork P, Bowler C, de Vargas C, Raes J, et al. (2011). A holistic approach to marine eco-systems biology. PLoS Biol 9:e1001177.

[7] Kelk SM, Olivier BG, Stougie L \& Bruggeman FJ (2012). Optimal flux spaces of genome-scale stoichiometric models are determined by a few subnetworks. Sci Rep 2, 580 .

[8] Klitgord N \& Segrè D. (2011). Ecosystems biology of microbial metabolism. Curr Opin Biotechnol 22:541-546.

[9] Klitgord, N \& Segrè, D (2010) The importance of compartmentalization in metabolic flux models: yeast as an ecosystem of organelles., Genome Informatics. International Conference on Genome Informatics 22: 41-55.

[10] Mintz-Oron S, Meir S, Malitsky S, Ruppin E, Aharoni A \& Shlomi T. (2012). Reconstruction of Arabidopsis metabolic network models accounting for subcellular compartmentalization and tissue-specificity. Proc Natl Acad Sci USA 109:339?344. 
[11] Monk J, Nogales J, Palsson BØ. (2014). Optimizing genome-scale network reconstructions. Nat Biotechnol 32:447-452.

[12] Müller AC, Bockmayr A. (2013). Flux modules in metabolic networks. J Math Biol.

[13] Raes J, Letunic I, Yamada T, Jensen LJ, Bork P. (2011). Toward molecular trait-based ecology through integration of biogeochemical, geographical and metagenomic data. Mol Syst Biol 7:473.

[14] Shoaie S, Karlsson F, Mardinoglu A, Nookaew I, Bordel S, Nielsen J. (2013). Understanding the interactions between bacteria in the human gut through metabolic modeling. Sci Rep 3:2532.

[15] Schellenberger J, Park JO, Conrad TM, Palsson BØ. (2010). BiGG: a Biochemical Genetic and Genomic knowledgebase of large scale metabolic reconstructions. BMC Bioinformatics $11: 213$.

[16] Stolyar S, Van Dien S, Hillesland KL, Pinel N, Lie TJ, Leigh JA, et al. (2007). Metabolic modeling of a mutualistic microbial community. Mol Syst Biol 3:92.

[17] Taffs R, Aston JE, Brileya K, Jay Z, Klatt CG, McGlynn S, Mallette N, Montross S, Gerlach R, InskeepWP, Ward DM, Carlson RP. (2009). In silico approaches to study mass and energy flows in microbial consortia: a syntrophic case study. BMC Systems Biology 3:114

[18] Thiele I, Heinken A, Fleming RMT. (2013). A systems biology approach to studying the role of microbes in human health. Curr Opin Biotechnol 24:4-12.

[19] Tzamali E, Poirazi P, Tollis IG, Reczko M. (2011). A computational exploration of bacterial metabolic diversity identifying metabolic interactions and growth-efficient strain communities. BMC Syst Biol 5:167.

[20] Wintermute EH, Silver PA. (2010). Emergent cooperation in microbial metabolism. Mol Syst Biol 6:407.

[21] Zengler K, Palsson BØ. (2012). A road map for the development of community systems (CoSy) biology. Nat Rev Microbiol 10:366372. 
[22] Zomorrodi, AR, \& Maranas, CD (2012). OptCom: a multi-level optimization framework for the metabolic modeling and analysis of microbial communities. PLoS Computational Biology, 8(2), e1002363. doi:10.1371/journal.pcbi.1002363

[23] Zomorrodi AR, Islam MM, Maranas CD. (2014). d-OptCom: Dynamic Multi-level and Multi-objective Metabolic Modeling of Microbial Communities. ACS Synth Biol 3:247?257. 


\section{Supplementary materials}

\subsection{Flux Modules are biologically relevant: illustration on modules for $E$. coli metabolic network in aerobic and anaerobic growth conditions}

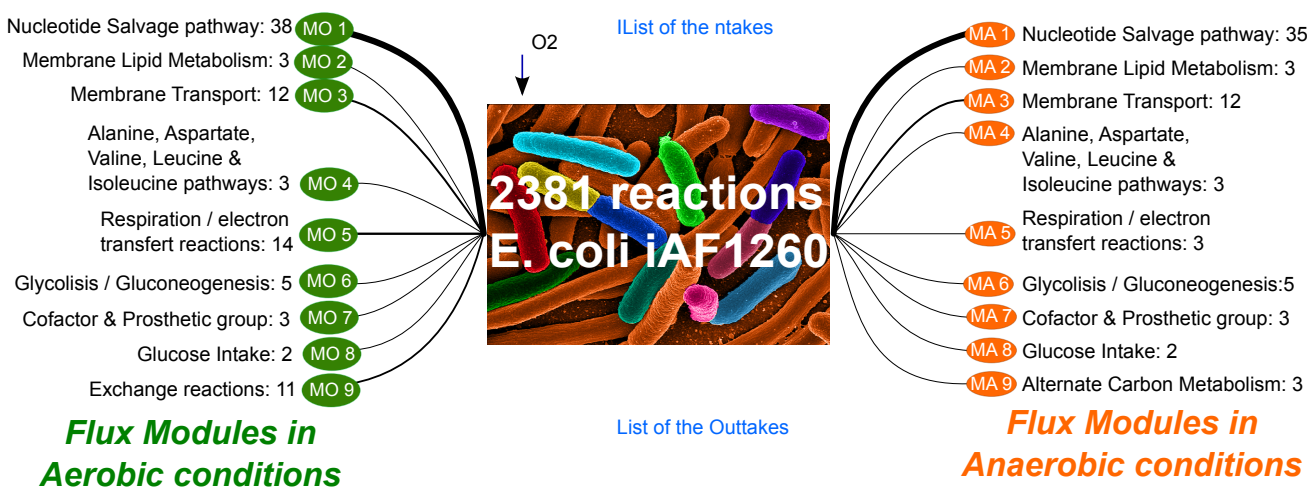

Above figure depicts modules of E. coli in both aerobic and anaerobic growth conditions. The metabolic model composed of 2381 reactions is analyzed in aerobic (left) and anaerobic conditions (right). For each conditions, one extracts 9 modules that are composed of distinct numbers of reactions (line width is proportional). Each module is associated to the pathways in which the flux module reactions are involved. Modules are in accordance to biological conditions. When challenged by an oxidative stress, most of flux modules of $E$. coli are conserved, except exchange reaction, respiration \& electron transfert, alternate carbon metabolism, which is in accordance to physiological knowledge. To a lesser extent, nucleotide salvage pathway is impacted by oxygen growth conditions.

\subsection{Metabolic modules of a methanogenic microbial system composed of Desulfovibrio vulgaris and Methanococcus maripaludis}

The metabolic model of $D$. vulgaris contains 145 reactions [22], whereas $M$. maripaludis model is composed of 97 reactions [16]. In order to link both strains within a $\mathrm{MCH}$ model, we duplicated exchange reactions of $\mathrm{H}_{2}$ in order to import/export the metabolite with either the other microorganism or environment. A similar procedure was done for Formate, Acetate and $\mathrm{CO}_{2}$, which overall introduces 12 exchange reactions. Finally, the whole ecosystem biomass was design to fit biomass functions of $D$. vulgaris and $M$. maripaludis as already 
published, while maintaining a respective proportion of 2:1 for both strains. $\mathrm{SCH}$ model of this community consist in merging both metabolic networks and removing all replicated reactions for considering one unique representative reaction. As results, SCH model is composed of 221 unique reactions, as a reduction of 243 reactions $\mathrm{MCH}$ model (respectively 145 and 97 reactions for D. vulgaris and M. maripaludis).
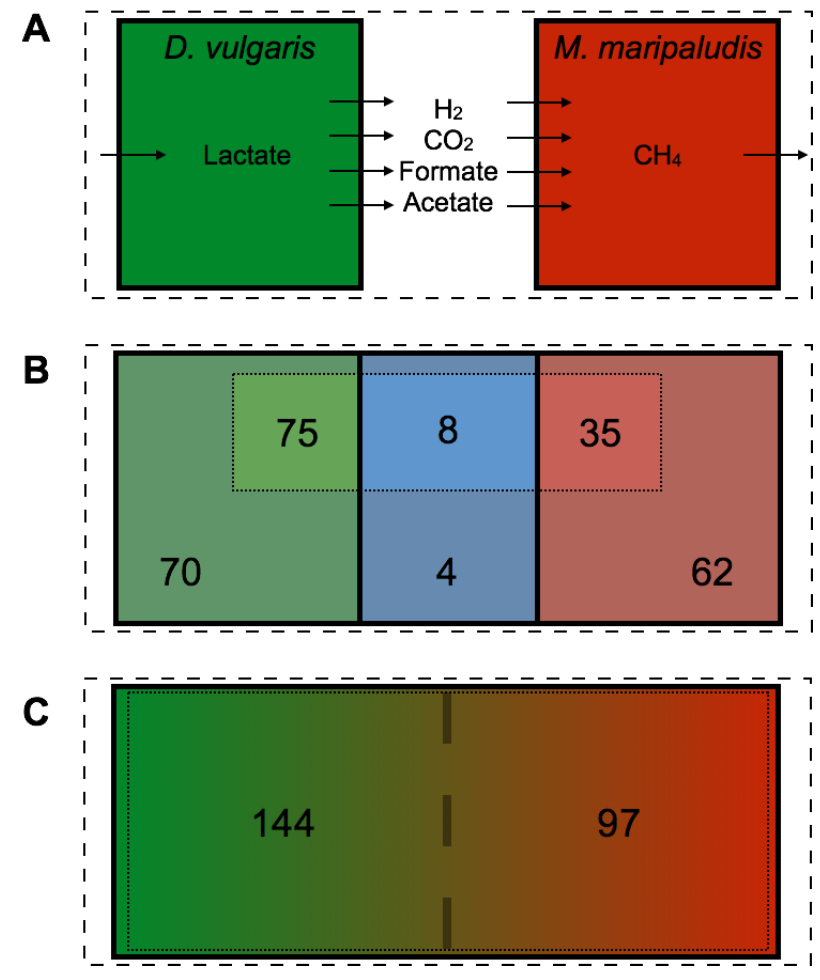

The SCH presents a disadvantage regarding the interplay between interchange fluxes; in this case, acetate and $\mathrm{H}_{2}$ are major players of electron transfer in anaerobic systems which role is an active area of research. Besides, for these two systems, the SCH links the fluxes of pentose phosphate system which could impact interpretations in future in silico developments 